\title{
Talla final de mujeres de Córdoba, Argentina, y exploración de la tendencia secular (1978-1988)
}

\author{
Fernando Agrelo, ${ }^{2}$ Laura Rosa Pascual, ${ }^{3}$ Beatriz Lobo ${ }^{2}$ \\ y Jacobo Sabulsky ${ }^{3}$
}

RESUMEN Este trabajo tuvo por objeto aportar datos locales sobre la talla final de mujeres de Córdoba, Argentina, y explorar la posibilidad de una tendencia secular en su crecimiento. Se examinaron 513 mujeres de 18 a 40 años de edad, madres de niños incluidos en el estudio CLACYD (Córdoba: lactancia, alimentación, crecimiento y desarrollo). Las mediciones se realizaron según técnicas estandarizadas, durante mayo y junio de 1994. Usando como normas de referencia datos del Centro Nacional de Estadísticas de Salud de los Estados Unidos de América, la media de la talla final de la población estudiada $(157,9 \mathrm{~cm})$ correspondió a una desviación estándar (DE) de $-0,97$ con respecto al percentil 50 de la norma de referencia de ese centro; la del grupo de estrato social alto $(159,7 \mathrm{~cm})$, a una DE de -0,67 y la del estrato muy bajo $(156,2 \mathrm{~cm})$, a una DE de $-1,25$. La diferencia entre los valores de los estratos sociales fue estadísticamente significativa $(\mathrm{P}<0,001)$. De la población estudiada, 2,3\% (6,4\% del estrato social muy bajo) registró tallas menores de $145 \mathrm{~cm}$. Para explorar la posibilidad de una tendencia secular, los datos resultantes se categorizaron en dos grupos de acuerdo con la edad materna en el momento del examen antropométrico: $G 1$ ( $\bar{x}: 24$ años) y $G 2$ ( $\bar{x}: 34$ años). Las mujeres del primer grupo registraton una talla final 0,4 cm mayor que la observada en las del segundo grupo $(\mathrm{P}=0,47)$. El aumento secular registrado es notoriamente inferior a los publicados en relación con otras provincias argentinas (1,2 y 1,4 cm/decenio) y algo menor al promedio que se notifica en estudios de poblaciones en Australia, Bélgica, los Estados Unidos, Japón y Noruega (0,6 cm/decenio). Se concluye que en el período analizado (1978-1988) las condiciones de vida en la ciudad de Córdoba no han mejorado de forma que se refleje en un aumento significativo de la talla de las mujeres adultas. Se recomienda que los programas de salud y nutrición materna concentren sus recursos en las madres de estrato social muy bajo que registran tallas inferiores a $145 \mathrm{~cm}$.

1 Este artículo se basa en el estudio CLACYD (Córdoba: lactancia, alimentación, crecimiento y desarrollo) subsidiado por la Organización Panamericana de la Salud, el Consejo de Investigaciones Científicas y Tecnológicas de la Provincia de Córdoba y la Secretaría de Ciencia y Tecnología de la Universidad Nacional de Córdoba. El estudio recibió apoyo financiero de la Fundación ARCOR.

2 Ministerio de Salud de la Provincia de Córdoba, Departamento de Maternidad e Infancia, División de Crecimiento y Desarrollo. Toda correspondencia debe dirigirse a F. Agrelo a la siguiente dirección postal: Arq. Thays 29, Barrio Nueva Córdoba, Caja postal 5 000, Córdoba, Argentina. E-mail: jsabuls@eco. uncor.edu
En el ser humano, al completarse el desarrollo del esqueleto cesa el crecimiento lineal y el adulto joven llega a su talla máxima (1). Esa talla resume la historia socionutricional de individuos y poblaciones. Según J. M. Tanner, "existe evidencia de que la diferencia

\footnotetext{
3 Universidad Nacional de Córdoba, Facultad de Ciencias Médicas, Córdoba, Argentina.
}

en la altura entre clases sociales en la población adulta se mantiene vigente por un sistema de movilidad social que, por alguna razón, produce un movimiento medio de las personas altas hacia arriba y de las personas más bajas hacia abajo" (2). El deterioro de la talla adulta proviene de múltiples inhibiciones ambientales que obstaculizan la expresión del potencial genético a lo largo de generaciones. 
Distintos estudios, entre ellos el que realizaron Martorell et al. (3) en el Instituto de Nutrición de Centro América y Panamá (INCAP), muestran que el retraso del crecimiento en edad temprana conduce a un tamaño corporal pequeño en el adulto. Las pérdidas causadas por un crecimiento insuficiente antes de los 3 a 5 años de vida no se recuperan más tarde, si bien algunos autores señalan que puede haber una recuperación parcial (4).

Este tema es de relevancia especial en el campo de la salud maternoinfantil, sobre todo en los países subdesarrollados. Como es bien sabido, la talla junto con otros parámetros antropométricos es de gran utilidad para reconocer a las mujeres en riesgo nutricional y vigilar los problemas que puedan surgir en torno a hijos de bajo peso al nacer, riesgos de mortalidad perinatal, neonatal e infantil, duración de la lactancia y complicaciones obstétricas (5-6).

En épocas anteriores, la talla final no se alcanzaba hasta los 25 años o aun más tarde. Actualmente, en poblaciones bien nutridas, la mayoría de los adultos alcanzan su potencial de máximo crecimiento lineal alrededor de los 18 años. Varios estudios de poblaciones europeas indican un logro de la talla máxima a los 16 o 17 años en las mujeres y a los 18 o 19 años en los varones (1-7). Este movimiento hacia la obtención de una talla adulta a edades más tempranas se registra en el complejo proceso denominado tendencia secular del crecimiento. El fenómeno se observa en períodos largos, por lo general de varios decenios. Los cambios seculares pueden ser positivos (aumentos, aceleraciones) o negativos (disminuciones, desaceleraciones). La tendencia secular no ha seguido un curso rectilíneo sino más bien una curva ondulante que se extiende a través de los siglos $(1,7)$. Es probable que se hayan producido disminuciones seculares marcadas en el tiempo de la revolución industrial, como los retrocesos que se han registrado durante las dos guerras mundiales señalados en los estudios efectuados en niños británicos (7).

En la mayor parte de los países se han originado cambios seculares positivos, asociados, según opinan distin- tos autores, con el mejoramiento de las condiciones de vida. Se sugiere una multiplicidad de causas; por ejemplo, mayor disponibilidad de nutrientes, mejoras en el nivel de salud, los procesos de urbanización e industrialización y la reducción en el tamaño de la familia (8-10). No obstante, todavía no hay una explicación completa del fenómeno. Algunos autores consideran que, por su naturaleza, la tendencia secular se debe casi enteramente a variaciones ambientales. En una hipótesis se propone que los cambios seculares obedecen en gran medida a la eliminación de factores que inhiben el crecimiento y no a la introducción de factores que lo estimulan (7). Hay quienes opinan que puede haber además una explicación genética: el aumento de heterosis debida a mayor exogamia $(1,2)$. En aquellos países donde se ha producido una tendencia secular marcada, los aumentos de talla y peso se han observado en todas las clases sociales (7).

Datos procedentes de Europa y los Estados Unidos de América señalan que, desde 1900 hasta 1975, la talla en el grupo de 5 a 7 años de edad ha aumentado de 1 a $2 \mathrm{~cm}$ por decenio y en el grupo de 10 a 14 años, de 2 a 3 cm por decenio. La menarca se ha adelantado cerca de 4 meses en cada período de 10 años. La tendencia ascendente ha sido menor en la talla adulta, pero se considera que desde 1880 hasta 1960 ha habido un incremento a razón de $0,6 \mathrm{~cm}$ por decenio $(2,8)$.

Tanto en América Latina como en la Argentina en particular, es escasa la información pertinente. Un estudio de Lejarraga et al. indica que desde 1938 hasta 1986 en los adolescentes de la ciudad de Buenos Aires se observó una tendencia secular al aumento de la talla de 1,7 y 1,2 cm por decenio, en varones y mujeres respectivamente (9). Desde 1950 se han observado aumentos de talla similares de 1,7 y $1,4 \mathrm{~cm}$ en varones y mujeres de la provincia de Entre Ríos (9). Por su parte, Cusminsky ha comparado estudios realizados entre 1922 y 1973 y describe incrementos seculares de peso y talla en niños de 6 a 12 años residentes en la Capital Federal y en la ciudad de La Plata (10).
Aquí se presentan los resultados de un trabajo que tuvo por objeto aportar datos locales sobre la talla final de mujeres de la ciudad de Córdoba, Argentina, y analizar, en una primera aproximación al tema, la tendencia secular que se observa en ese parámetro.

\section{MATERIALES Y MÉTODOS}

Se utilizó la base de datos del estudio CLACYD (Córdoba: lactancia, alimentación, crecimiento y desarrollo) que se inició en 1993 con el propósito principal de analizar las características de la alimentación, el crecimiento y el desarrollo de una cohorte representativa de niños de la ciudad de Córdoba, Argentina, durante sus primeros 5 años de vida. Las principales características de ese estudio - la estrategia metodológica prospectiva, los criterios de selección de la muestra y otrasaparecen descritas en otros trabajos publicados $(11,12)$.

De los parámetros antropométricos registrados en el estudio CLACYD, el presente trabajo analiza solamente la talla de las madres participantes, que fue registrada durante mayo y junio de 1994, cuando los niños cumplieron un año de edad. El examen antropométrico de las madres se llevó a cabo en esas fechas porque ya había pasado un tiempo aceptable desde el nacimiento del niño. Es decir, de este modo se evitaron los efectos del peso agregado del embarazo en la compresión de los discos intervertebrales y la distorsión postural concomitante (5). Se calculó la edad de la madre en el momento del examen por el registro de la fecha de nacimiento que figuraba en la historia clínica de la maternidad y las cifras se redondearon según los años cumplidos. Los resultados que presentamos corresponden a 513 madres de 18 a 40 años de edad.

Las tallas fueron medidas por profesionales previamente capacitados según las técnicas e instrumentos recomendados por Lohmann et al. (13). Las mediciones se estandarizaron según el procedimiento diseñado por Habicht (14). Durante la medición, las madres estaban descalzas o llevaban medias 
finas y vestían ropa liviana que permitiera observar adecuadamente la posición corporal $(5,13)$.

La población del estudio CLACYD estaba clasificada en seis estratos sociales definidos según la ocupación de la persona principal responsable del sustento familiar (11). Para efectos del estudio aquí presentado, se hizo un análisis comparativo de los dos estratos sociales ubicados en los dos extremos del sistema de clasificación, que incluían los grupos ocupacionales siguientes: estrato alto (A): empleadores, profesionales, técnicos terciarios y personal jerárquico, y estrato muy bajo (B): trabajadores temporarios de baja calificación; por ejemplo, vendeores ambulantes, empleadas domésticas, peones de la construcción y maleteros.

Con objeto de explorar la posibilidad de un cambio secular, los datos se dividieron en dos grupos en función de la edad materna en el momento del examen antropométrico (cuadro 1). El procesamiento de los datos se realizó con el programa estadístico SPSS versión 5 (15). Se calcularon las medias $(\bar{x})$, los errores estándar y los percentiles. Se describe en particular el percentil 10 porque es el punto de corte empleado generalmente para identificar los valores antropométricos atípicos. Para analizar las diferencias entre los promedios de las tallas por estrato social y por grupos de edad, se empleó la prueba $t$ de Student. Se consideraron estadísticamente significativas las di-

CUADRO 1. Distribución de una población de madres, según la edad, en el momento en que se les administró un examen antropométrico. Córdoba, Argentina, 1994

\begin{tabular}{ccc}
\hline Grupo & $\begin{array}{c}\text { Edad } \\
(\text { años })\end{array}$ & $\begin{array}{c}\text { Año en que se alcanzó } \\
\text { en promedio } \\
\text { la talla final }\end{array}$ \\
\hline $1(n: 344)$ & $\begin{array}{c}18 \text { a } 29 \\
(\overline{\mathrm{x}}: 24)\end{array}$ & 1988 \\
$2(n: 169)$ & $\begin{array}{c}30 \text { a } 40 \\
(\overline{\mathrm{x}}: 34)\end{array}$ & 1978 \\
& $\begin{array}{c}18 \text { a } 40 \\
\text { Total }(513)\end{array}$ & 1985 \\
& $(\overline{\mathrm{x}}: 27)$ & \\
\hline
\end{tabular}

CUADRO 2. Medias $(\bar{x})$, errores estándar (EE) y percentiles de talla final (cm) de mujeres de la ciudad de Córdoba, Argentina, 1994

\begin{tabular}{|c|c|c|c|c|c|c|c|c|c|}
\hline & \multirow[b]{2}{*}{$\bar{x}$} & \multirow[b]{2}{*}{ EE } & \multicolumn{7}{|c|}{ Percentil } \\
\hline & & & 3 & 10 & 25 & 50 & 75 & 90 & 97 \\
\hline $\begin{array}{l}\text { Población total } \\
\qquad(n: 513)\end{array}$ & 157,9 & 6,1 & 145,8 & 150,2 & 154,0 & 157,7 & 162,0 & 165,6 & 169,5 \\
\hline $\begin{array}{l}\text { Estrato social } A^{a} \\
\qquad(n: 93)\end{array}$ & $159,7^{b}$ & 5,9 & 149,2 & 152,2 & 155,2 & 160,0 & 163,2 & 166,8 & 174,5 \\
\hline $\begin{array}{l}\text { Estrato social } \mathrm{B}^{\mathrm{c}} \\
\qquad(n: 110)\end{array}$ & 156,2 & 6,4 & 142,5 & 146,6 & 151,9 & 156,1 & 161,4 & 165,0 & 167,3 \\
\hline
\end{tabular}

ferencias con valor $P$ menor de $0,05(P$ $<0,05)$.

\section{RESULTADOS}

La muestra inicial del estudio CLACYD en 1993 fue de 709 niños. En 1994 se realizó un examen antropométrico de 560 madres de esos niños, o sea, de $79 \%$ de la muestra inicial. Cabe señalar que las pérdidas durante el seguimiento —que se produjeron principalmente debido a cambios de domicilio y, en muy pequeña proporción, por rechazo a participar en el estudio-no modificó significativamente la distribución original de la población en estratos sociales. Se excluyeron del análisis 36 mujeres menores de 18 años, 9 mayores de 40 años y 2 otras de las que se desconocía la edad. Se excluyeron las menores de 18 años porque algunas de ellas (maduradoras lentas) podían no haber alcanzado la talla final en el momento del examen; y las mayores de 40 años, para evitar el riesgo de incorporar algunos casos en los que la disminución de la talla adulta hubiera comenzado más tempranamente que lo habitual (16). Por lo tanto, los resultados que presentamos corresponden a 513 madres de 18 a 40 años de edad.

En el cuadro 2 se observa que los valores medios de talla de los estratos sociales (A) y (B) corresponden a 1,8 cm por encima y $1,7 \mathrm{~cm}$ por debajo de la media correspondiente a la población total. La diferencia entre ambos estratos $(3,5 \mathrm{~cm})$ es estadísticamente significativa $(P<0,001)$. El error estándar es similar en los dos grupos, pero algo mayor en el de estrato muy bajo. Al igual que en los promedios, las diferencias por estrato social a nivel del percentil 10 son marcadas. El valor del estrato muy bajo se sitúa en los 146,6 $\mathrm{cm}$, es decir 5,6 y 3,6 cm por debajo de los valores correspondientes al estrato alto y a la población total, respectivamente. Además, 146,6 cm representa un valor muy cercano al que se acepta generalmente para definir la categoría de alto riesgo $(145 \mathrm{~cm})$. De la población, 2,3\% (6,4\% del estrato social muy bajo) registró tallas inferiores a $145 \mathrm{~cm}$.

En la figura 1 se presentan los valores medios de nuestro estudio con respecto al percentil 50 de la norma de referencia del NCHS (17). La media de la población estudiada corresponde a $-0,97 \mathrm{DE}$, el estrato alto a $-0,67 \mathrm{DE}$ y el estrato bajo a $-1,25$ DE de la norma citada.

En el cuadro 3 se comparan las medias de la talla final correspondientes a dos grupos de edad (véase el cuadro 1), con objeto de explorar la posibilidad de detectar una tendencia secular. Los datos muestran que las mujeres que alcanzaron en promedio la talla final en 1988 (grupo 1) registraron una talla $0,4 \mathrm{~cm}$ mayor que la observada en mujeres que alcanzaron en promedio la talla final 10 años antes, es decir en 1978 (grupo 2), $(P=0,47)$. 
FIGURA 1. Medias de la talla final $(\mathrm{cm})$ de la población de madres estudiada expresadas en puntuaciones estandarizadas $(Z)$ de la norma de referencia (NCHS $\left.{ }^{a}\right)$. Córdoba, Argentina, 1994

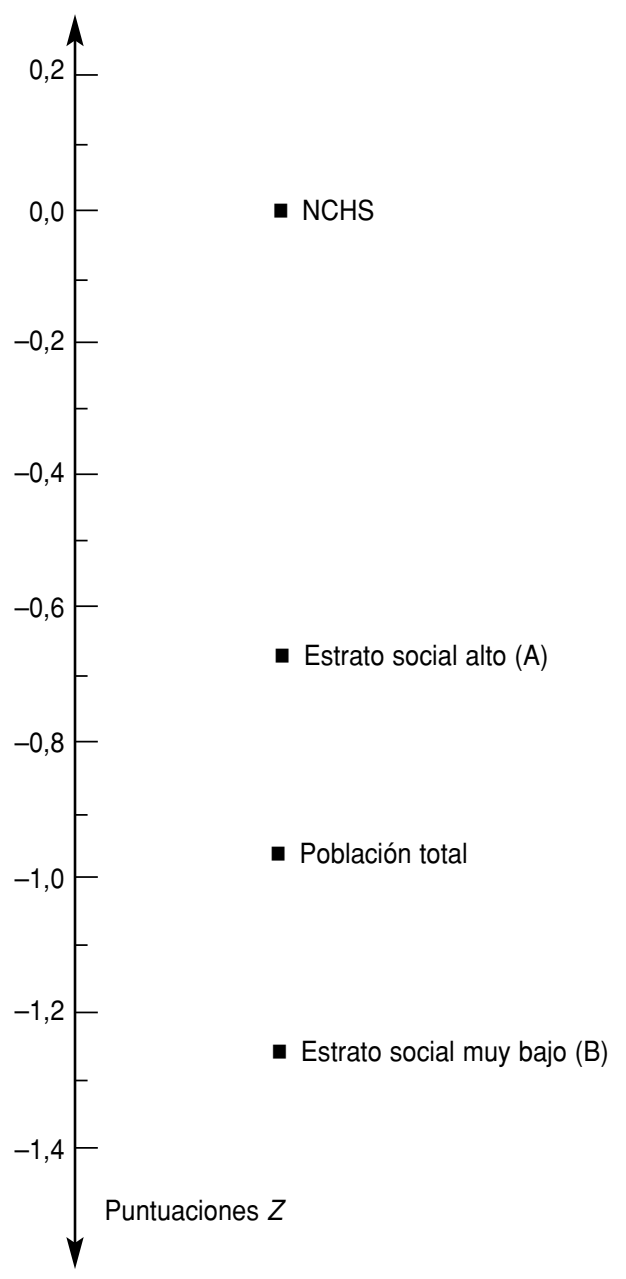

${ }^{\text {a }} \mathrm{NCHS}=$ National Center for Health Statistics [Centro Nacional de Estadísticas de Salud], Estados Unidos de América.

CUADRO 3. Exploración de la tendencia secular en la talla final de la población de madres estudiada. Córdoba, Argentina, 1994

\begin{tabular}{lcccc}
\hline Grupo & $\begin{array}{c}\text { Año en que el promedio } \\
\text { del grupo alcanzó la talla final }\end{array}$ & $\begin{array}{c}\bar{x}^{\mathrm{a}} \\
(\mathrm{cm})\end{array}$ & IC95\% b & $P$ \\
\hline $1(n: 344)$ & 1988 & 158,0 & 156,8 a 159,2 & 0,47 \\
$2(n: 169)$ & 1978 & 157,6 & 155,8 a 159,5 & \\
\hline $\begin{array}{l}\text { a } \bar{x}=\text { media. } \\
\text { b IC95\% = Intervalo de confianza de } 95 \% .\end{array}$ & & & &
\end{tabular}

\section{DISCUSIÓN}

El cuadro 2 muestra que los valores promedio de la talla final y la distribución en percentiles son notablemente
Dado que la talla adulta es el resultado final de todo el proceso de crecimiento, para conocer la historia socionutricional de las mujeres estudiadas deberíamos hacer referencia al estrato social de sus familias de origen, información que no hemos registrado. Los resultados se han analizado sobre la base del estrato social al que pertenecían en el momento del examen antropométrico, lo cual, aun con limitaciones, permite resumir de algún modo la brecha existente entre condiciones sociales disímiles. $\mathrm{Al}$ parecer, esta brecha persiste aun en países industrializados. Tanner ha señalado que hay una diferencia de $2 \mathrm{~cm}$ entre la talla media de los grupos privilegiados y la de los trabajadores no calificados de Inglaterra (18).

En este trabajo confrontamos solamente los estratos alto y muy bajo porque entendemos que en ellos la movilidad social es escasa o inexistente. Es muy probable que las condiciones de vida en el momento del examen antropométrico fueran iguales a las que existían desde que las madres del estudio eran niñas.

Al comparar nuestros resultados con los del trabajo de Lejarraga et al. sobre adolescentes argentinos (9), llama la atención que los promedios de talla final detectados por esos investigadores para todo el país $(160,4 \mathrm{~cm})$ y para la provincia de Córdoba en particular $(159,8 \mathrm{~cm})$ son muy similares a los valores correspondientes al estrato alto de nuestra población $(159,7 \mathrm{~cm})$. Sin embargo, esto puede deberse a que la muestra de Lejarraga et al. no es representativa de toda la población de adolescentes de la Argentina, ya que $77 \%$ pertenecen a los estratos sociales medios y altos. Los datos del estudio CLACYD corresponden a una muestra representativa del conjunto de mujeres madres de nuestro medio, pues abarca a todos los nacimientos registrados en un lapso de 12 días en instituciones pública y privadas, aproximadamene $98 \%$ de los niños nacidos en la ciudad de Córdoba en ese período. Dadas las características de la muestra, pensamos que los resultados obtenidos tienen suficiente valor epidemiológico para emplearse en programas orienta- 
dos a la salud y nutrición materna. Si bien se recomienda establecer localmente los puntos de corte para lograr un máximo de sensibilidad y especificidad $(5,6)$, por lo general se acepta que las mujeres con tallas menores de $145 \mathrm{~cm}$ están en la categoría de alto riesgo (19). En nuestro estudio, las mujeres pertenecientes al estrato social muy bajo se encontraban en esa situación. Por lo tanto, los programas deberían concentrar sus recursos en ese sector de población de mayor riesgo.

Nuestros resultados muestran una tendencia secular de un aumento de la talla final de $0,4 \mathrm{~cm}$ en las mujeres (cuadro 3). A fin de corroborar o contradecir la tendencia, comparamos las medias de la talla final de la población estudiada con los resultados presentados por Sabulsky (20). Este último trabajo, orientado a describir los perfiles diferenciales del retraso del crecimiento en escolares de la ciudad de Córdoba, se realizó en una muestra aleatoria de 1453 niños que en 1985 cursaban el primer grado. La talla materna se incluyó entre las medidas antropométricas registradas por técnicas estandarizadas. Las madres de esos niños alcanzaron en promedio su talla final en 1970 (véase el cuadro 3). Al cotejar la media de la talla final informada por Sabulsky $(157,3 \mathrm{~cm})$ con la que aquí registramos $(157,9 \mathrm{~cm})$, se observa una diferencia de 0,6 cm. Es decir, las madres de nuestro estudio, que alcanzaron en promedio su talla final 15 años después que las integrantes del trabajo de Sabulsky, tuvieron una talla final media $0,6 \mathrm{~cm}$ más alta $(P$ $=0,07)$, lo cual corrobora la tendencia secular de un aumento de la talla de 0,4 $\mathrm{cm} /$ decenio, como indican nuestros resultados. Los datos de Sabulsky, al igual que los de CLACYD, corresponden a muestras representativas de madres de los distintos estratos sociales de la ciudad de Córdoba. En ambos estudios se empleó una metodología antropométrica estandarizada, que también hace posible comparar los resultados.

En la Argentina, Lejarraga et al. (9) informaron de cambios seculares positivos superiores a los de nuestro estudio en mujeres adolescentes: 1,2 cm/ decenio en la ciudad de Buenos Aires desde 1938 y 1,4 cm/decenio en Entre Ríos a partir de 1950. Como se ha expresado, las diferencias en los resultados obedecen posiblemente a que las muestras seleccionadas son disímiles, representativas de todos los estratos sociales en nuestro caso y con un sesgo social hacia los niveles medios y altos en el estudio de adolescentes citado. Con respecto a la situación en otros países, no hemos encontrado trabajos sobre América Latina en la bibliografía consultada. Meredith, al analizar datos de Australia, Bélgica, Estados Unidos, Japón y Noruega, de este siglo y del siglo pasado, informó que en períodos de 40, 100 o aun más años, se ha registrado en promedio una tendencia secular positiva en la talla adulta de 0,6 $\mathrm{cm}$ por decenio (8). Las encuestas realizadas en Holanda entre 1965 y 1980 mostraron que la talla de las mujeres había aumentado a razón de 0,8 $\mathrm{cm} /$ decenio (24). En el Japón, el aumento de talla en las mujeres fue de $1,5 \mathrm{~cm} /$ decenio entre 1957 y 1967 y 1,2 cm/decenio entre 1967 y 1977 (21). Tanner (16) informó que hubo un incremento promedio de $1,25 \mathrm{~cm} /$ decenio en la talla adulta de varones ingleses nacidos entre 1900 y 1946, y de $0,6 \mathrm{~cm} /$ decenio entre los que nacieron desde 1946 hasta 1960. En estratos sociales altos de la India, al comparar las medias de las tallas de madres mayores de 40 años con las de sus hijas de 18 años, se detectó una tendencia secular positiva de $0,82 \mathrm{~cm}$, o sea un aumento de $0,4 \mathrm{~cm} /$ decenio (22).

\section{REFERENCIAS}

1. Falkner F. Consideraciones sobre el desarrollo humano. En: Falkner F. Desarrollo humano. Barcelona: Salvat; 1969. pp. 12-47.
2. Tanner JM. Crecimiento postnatal. En: Mitchell RG. Crecimiento y desarrollo del niño Barcelona: Jims; 1975. pp. 169-223.
En suma, se concluye que la media de la talla final de la población estudiada correspondió a $-0,97 \mathrm{DE}$ de la norma de referencia del Centro Nacional de Estadísticas de Salud de los Estados Unidos y la diferencia entre los estratos sociales alto y muy bajo fue estadísticamente significativa. De la población estudiada, 2,3\% (6,4\% del estrato social muy bajo) tuvieron tallas menores de $145 \mathrm{~cm}$. Se observó una tendencia secular de un aumento aproximado de $0,4 \mathrm{~cm} /$ decenio, valor decididamente más bajo que los que se mencionan en los estudios de otras provincias argentinas y algo menor que el valor promedio que indican los datos de Australia, Bélgica, Japón, los Estados Unidos y Noruega. Este resultado sugiere que en el período analizado (1978-1988) no ha habido un mejoramiento en las condiciones de vida de la población de la ciudad de Córdoba que se refleje en el aumento significativo de la talla de las mujeres adultas.

Reiteramos que la población estudiada constituyó una muestra representativa de las mujeres/madres de la ciudad de Córdoba. A fin de confirmar nuestros hallazgos y poder generalizarlos a la población de mujeres adultas de la ciudad de Córdoba, se requieren estudios locales actualizados cada 10 o 15 años, basados en muestras amplias y representativas de todas las mujeres de nuestro medio. Dichos estudios permitirán analizar la evolución de los cambios seculares que hemos explorado en este trabajo.

Agradecimiento. Los autores expresan su agradecimiento a las familias que participaron en el estudio y a los profesionales que llevaron a cabo los exámenes antropométricos.
3. Martorell L, Rivera J, Kaplowitz H. Consecuencias del retraso en el crecimiento durante la primera infancia sobre la talla 
adulta en las zonas rurales de Guatemala. En: La nutrición infantil: consecuencias a largo plazo. Anales Nestlé 1993;48(1). pp. $45-54$.

4. Satyanarayana K, Swaminathan MC, Narasinga Rao BS. Effect of nutritional deprivation in early childhood on later growth-a community study without intervention. Am J Clin Nutr 1981;34: 1636-1637.

5. World Health Organization. Physical status: The use and interpretation of anthropometry. Geneva: WHO; 1995. (Technical report 854)

6. Maternal anthropometry for prediction of pregnancy outcomes: Memorandum from a USAID/WHO/PAHO Mother Care Meeting. Bull World Health Organ 1991; 69(5):523-532.

7. Roche A. Secular trends in stature, weight, human growth and maturation. En: Roche A. Secular trends in human growth, maturation and development. University of Chicago Press; 1979. pp. 3-27. (Monographs of the Society for Research in Child Development. Serial 179. Vol. 44 No. 3-4).

8. Meredith HV. Findings from Asia, Australia, Europe, and North America on secular change in mean height for children, youths and young adults. Am J Phys Anthrop 1976;44:315-326.

9. Lejarraga H, Meletti I, Biocca S, Alonso V. Peso y talla de 15214 adolescentes de todo el país: tendencia secular. Arch Arg Pediatr 1986;84:219-235.

10. Cuminsky M. Tendencia secular del crecimiento en la Argentina. Rev Hosp Niños 1978;20(80):197-200.

11. Sabulsky J, Batrouni L, Carballo R, Reyna S, Quiroga D, Roitter H, et al. Alimentación en el primer mes de vida, por estratos sociales, Córdoba, Argentina. Bol Oficina Sanit Panam 1995;119(1):15-27.

12. Sabulsky J, Batrouni L, Quiroga D, Reyna S, Carballo R, Zamora S, et al. Perfiles de lactancia al mes de vida. Arch Arg Pediatr 1995;93:151-157.

13. Lohmann TG, Roche AF, Martorell R, eds. Anthropometric standardization reference manual. Champaign, Illinois: Human Kinetics Books; 1988.

14. Habitch JP. Estandarización de métodos epidemiológicos cuantitativos sobre el terreno. Bol Oficina Sanit Panam 1974;76: 375-384.

15. Norusis MJ. Statistical package for the social sciences.(SPSS/PC+) version 5.0. Chicago, Illinois $60611 ; 1998$

16. Tanner JM, Goldstein $\mathrm{H}$, Whitehouse RH. Standards for children's height at ages 2-9 years allowing for height of parents. Arch Dis Child 1970:45:755-762.

17. National Center for Health Statistics (U.S.), United States Public Health Service. Height and weight of youths 12-17 years, United States. Washington, DC: Government Printing Office; January 1973. (Vital and Health Statistics. Series 11-N 119 DHEW Pub N (HSM) 73-1601).

18. Tanner JM. Growth as a measure of the nutritional and hygienic status of a population. Horm Res 1992;328(Suppl 1): 106-115.

19. Gopalan C. Women and nutrition in India: Some practical considerations. Bulletin of the Nutrition Foundation of India 1989;10(4):1-8.

20. Sabulsky J. Estudio socioepidemiológico de la desnutrición crónica en escolares de la ciudad de Córdoba (tesis doctoral). Universidad Nacional de Córdoba, Facultad de Ciencias Médicas; 1990.

21. Tanner JM. Growth as a mirror of the condition of society: Secular trends and class distinctions. Acta Paediatr Jpn 1987;29: 96-103.

22. Nutrition Foundation of India. Heights and weights of parents. En: Growth of affluent Indian girls during adolescence. New Delhi: NFI; 1989. pp. 17-23. (Scientific report 10)

Manuscrito recibido el 12 de abril de 1997 y aceptado para publicación en versión revisada el 9 de diciembre de 1998.
ABSTRACT

\section{Full adult height of women in Cordoba, Argentina, and an exploration of secular trends 1978-1988}

The objective of this work was to contribute local data concerning the full adult height of women in Cordoba, Argentina, and to explore the possibility of a secular trend in their heights. For the study, 513 women were examined during May and June 1994. All of the women were between 18 and 40 years of age and were mothers of children who were included in a study on lactation, feeding, growth, and development in Córdoba. The measurements were carried out applying standardized techniques and using as a reference standard the 50th-percentile level data from the U.S. National Center for Health Statistics. The mean full height of the Córdoba population studied was $157.9 \mathrm{~cm}, 0.97$ standard deviation (SD) below the reference norm. For the women from the highest of six socioeconomic strata, the mean height was $159.7 \mathrm{~cm}$ $(-0.67 \mathrm{SD})$; the mean for women from the lowest stratum was $156.2 \mathrm{~cm}(-1.25 \mathrm{SD})$. The difference in the means of those two socioeconomic groups was statistically significant $(P<0.001)$. Of the population studied, $2.3 \%(6.4 \%$ of the lowest social stratum $)$ were shorter than $145 \mathrm{~cm}$. In order to explore the possibility of a secular trend, the resulting data were categorized into two groups according to the mother's age at the time of the anthropometric examination, one group with a mean age of 24 and a second group with a mean age of 34 . The younger women had a mean adult height $0.4 \mathrm{~cm}$ greater than that of the older women $(P=0.47)$. This secular increase in height is notably smaller than that reported for other Argentine provinces (1.2 and $1.4 \mathrm{~cm} /$ decade) and somewhat lower than the average reported in population studies in Australia, Belgium, the United States, Japan, and Norway $(0.6 \mathrm{~cm} /$ decade $)$. The authors conclude that in the period analyzed, 1978-1988, the living conditions in the city of Córdoba have not improved in a way that is reflected in a significant increase in the height of adult women. The authors recommend that maternal health and nutrition programs concentrate their resources on the mothers from the lowest socioeconomic stratum who are shorter than $145 \mathrm{~cm}$. 